\title{
Calculating Trust Level of X.509 Certificates
}

\author{
Zakia El uahhabi, Hanan El bakkali \\ Information Security Research Team \\ ENSIAS - Mohammed V University \\ Rabat, Morocco
}

\begin{abstract}
The X.509 certificate is widely used by the organizations and people in order to confirm their identities in online transaction. It is created, signed, and issued by certificate authority (CA) following the procedures which are defined in a certificate policy. However, the deficiency in these procedures may create a trust lack in the certificate. Also, due to the CA failures or compromises, the risk of trusting a malicious certificate increase. Then, relying party $(R P)$ should verify certificate trustworthiness in order to accept it or not. In reality, it is difficult for $R P$ who hasn't the technical competences to judge it. In this context, he needs an automated mechanism for evaluating a certificate trust level. In this paper, we provide him this mechanism. In fact, we suggest a new architecture for calculating a certificate trust. A proposed algorithm is used by this architecture and takes as input three parameters that are the rating of certification fields content, the certificate policy quality, and the calculated CA trust level.
\end{abstract}

\section{Introduction}

Public Key Infrastructure (PKI) is an effective technology used for management and distribution of public keys and certificates in order to ensure the security of electronic communication and transaction in open environment. The trust propagation in this infrastructure depends on its syntactic trust structure which is known as a trust model [4]. PKI trust model permits to extend and manage trust relationships between different parties along a set of certificates providing a trust chain.

In X.509 PKI, certificate authority (CA) issues a certificate, which presents a proof for confirming its holder identity, by using its own rules that are defined in certificate policy (CP) and certification practice statement (CPS). In open network, a CA can be attacked and used to issue fraudulent certificates and false signature. So, trust level of these certificates comes into a question. Moreover, RP should verify certificate trustworthiness in order to accept it or not. He needs to verify its signature, certification path from the certificate to a root certificate, so on, Also, he needs to read a CP/CPS that the CA follows during the certificate life cycle. Practically, it is difficult for them to evaluate the CP/CPS which is technical and long. Consequently, RP needs an automated mechanism for making a trust decision about a received certificate. In this paper, we suggest this mechanism used for determining a certificate trust level.

The objective of our paper is to define a trust framework that will automatically calculate a trust level associated with certificate (TLoCERT). For instance, when RP receives a certificate issued by a specific CA, he needs to check its trustworthiness. In order to make a decision about whether to accept or not a received certificate, he requests from our system its TLoCERT.

We have contributed in suggesting an algorithm used by our proposed framework for computing a certificate trust level. This algorithm takes as input three parameters which are the certificate policy quality (CPQ) which evaluate the quality of the procedures announced in the published $\mathrm{CP}$, the computed CA trust level (TLOCA), and the rating of certificate fields content that define the initial assessment of certificate correctness. We will talk in details about its calculation method in Section V.

The rest of this paper is structured as follows. We briefly recall the notions and concepts of trust and reputation in the Section II. Section III presents some related work. Afterward, in Section IV, we describe our suggested trust framework architecture. Section V present our proposed trust level calculation algorithm. Then, we define in Section VI a scenario that illustrates how RP makes a decision about a certificate trustworthiness using our solution. The last section presents a conclusion and some of future works.

\section{Trust and Reputation Concepts}

Reputation plays a crucial role in the process of trust building. It is important to note that the concept of reputation is closely related to that of trust, but it is clear that they are different. Reputation can be considered as a collective measure of trustworthiness based on the referrals or ratings from other members in a community [2]. It is assessed by using a reputation system which aggregates these ratings. Moreover, a reputation value may be one important parameter that can be used to evaluate trust. In this paper, we measure a CA's trustworthiness which plays the trustee role. Our approach is also based on the measurement of CAs reputation. 
Trust and trustworthiness are generally confused, they are distinct concepts. Trustworthiness is a property or characteristic of an individual while trust is an attribute of an established relationship between exchange partners $[3,1]$. Both concepts help the user to make decisions and complement each other. In general, the most researchers define the trust concept in a particular context. In this paper, we present some definitions which can be applicable to e-services environment.

In [6], the authors define trust as the subjective expectation an agent has about another's future behavior based on the history of their encounters. Moreover, the definition of trust is related to party willingness to be vulnerable to the actions of another party based on the expectation that the other will perform a particular action important to the trustor, irrespective of the ability to monitor or control that other party [7].

Josang et al.[8] define trust as the extent to which a given party is willing to depend on something or somebody in a given situation with a feeling of relative security. In our approach, RPs make a decision about the certificate trustworthiness based on its trust level. Thus, our approach is based on this definition.

Clearly, the trustee reputation may influence decisions made by a trustor [4]. The reputation concept is closely related to that of trustworthiness and trust, but it is evident that they are distinct. According to Josang et al. [2], reputation is what is generally said or believed about a person's or thing's character or standing. It is based on the reviews or rating from member in a community and can be considered as a collective measure of trustworthiness [2]. The difference between trust and reputation can be illustrated by the following statements [8]:

1. "I trust you because of your good reputation."

2. "I trust you despite your bad reputation."

Statement 1 reflects that a relying party bases his trust on the reputation trustee. Statement 2 reflects that the RP has some private knowledge about the trustee which is based on various factors.

In this paper, we focus on determining the CA trust level which defines its trustworthiness based on the various factors such as a CA reputation. The calculated level is one of the parameters used for measuring the certificate trust level.

\section{Related work}

Many approaches are suggested for evaluating trust in PKI. In this regard, some approaches are discussed below. The authors in [9] propose a trust model for assessing trust in PKI. They introduce a trust calculus in order to propagate and derive trust among different entities in a certification chain. The proposed approach of $[10,11]$ defines a mechanism for evaluating a CA trustworthiness by using a distributed reputation system. This system aggregates the given user trust view, which presents the collected information about the CAs while browsing websites, in order to compute an issuer trust recommendation value. The calculated value is distributed to users for making decision about certificate trustworthiness. However, the suggested approach necessities a long time until a user have known all required CAs on the basis of their browser history.

In [12], the authors suggest a framework used for assessing a certificate risk level on the basis of certain trust characteristics and criteria. The proposed framework permits user to make decision whether accept or not a certificate for a particular transaction by evaluating its risk level. It includes three modules involved in a risk level calculation. The first module collects and stores an untrusted and trusted x.509 certificate, while the second module collects attribute/criteria which are taken into consideration in calculation process. The last module classifies a risk level in three values as low risk, medium risk, and high risk.

The authors of [13] propose a technique for measuring the CA trust level by evaluating the certificate issued by this authority. In their suggested model, a certificate authority trust service assess the collected certificates, which have been sent by users, according to the rules based on different factors such as certificate validity. However, the authors do not introduce a mechanism for checking if the collected certificates, which are submitted by clients, are malicious or not. Lucas et all presents in [14] an implementation of trust quantification and calculation in the X.509 PKI model. They introduce three category of trust in the X.509 PKI: PKI trust, policy trust, and authentication trust. Each category of trust is evaluated by a calculated trust value. This value is represented by using an ASN.1 structure and included in X.509 model in order to allow user to check the certificate trust level. In case of PKI trust, the authors consider that an end user defines a trust value according to his relationship with the trust anchor, but that makes his task more complex when he encounters unknown CA.

In our point of view, there are others factors that influence certificate trustworthiness as CA security risk that is defined in [4], CA reputation, CP quality, and certificate extension fields' content.

To the best of our knowledge, there is no proposed work that integrates all these factors for evaluating certificate trustworthiness. Then, we propose a new approach that integrates these factors in the assessment process of certificate trustworthiness. We will show more details about our work in next section. 


\section{Our suggested framework}

Our proposed approach suggests several steps for computing the TLOCERT. Figure 1 shows our suggested trust framework architecture. It comprises different components required in the TLOCERT computation. They are explained below.
- Security Module: It contains the following components: CA-SL Evaluator and SL DB. The evaluator retrieves from the SL DB some information such as EAL of CA software and its implemented security standard, in order to use it for assessing a

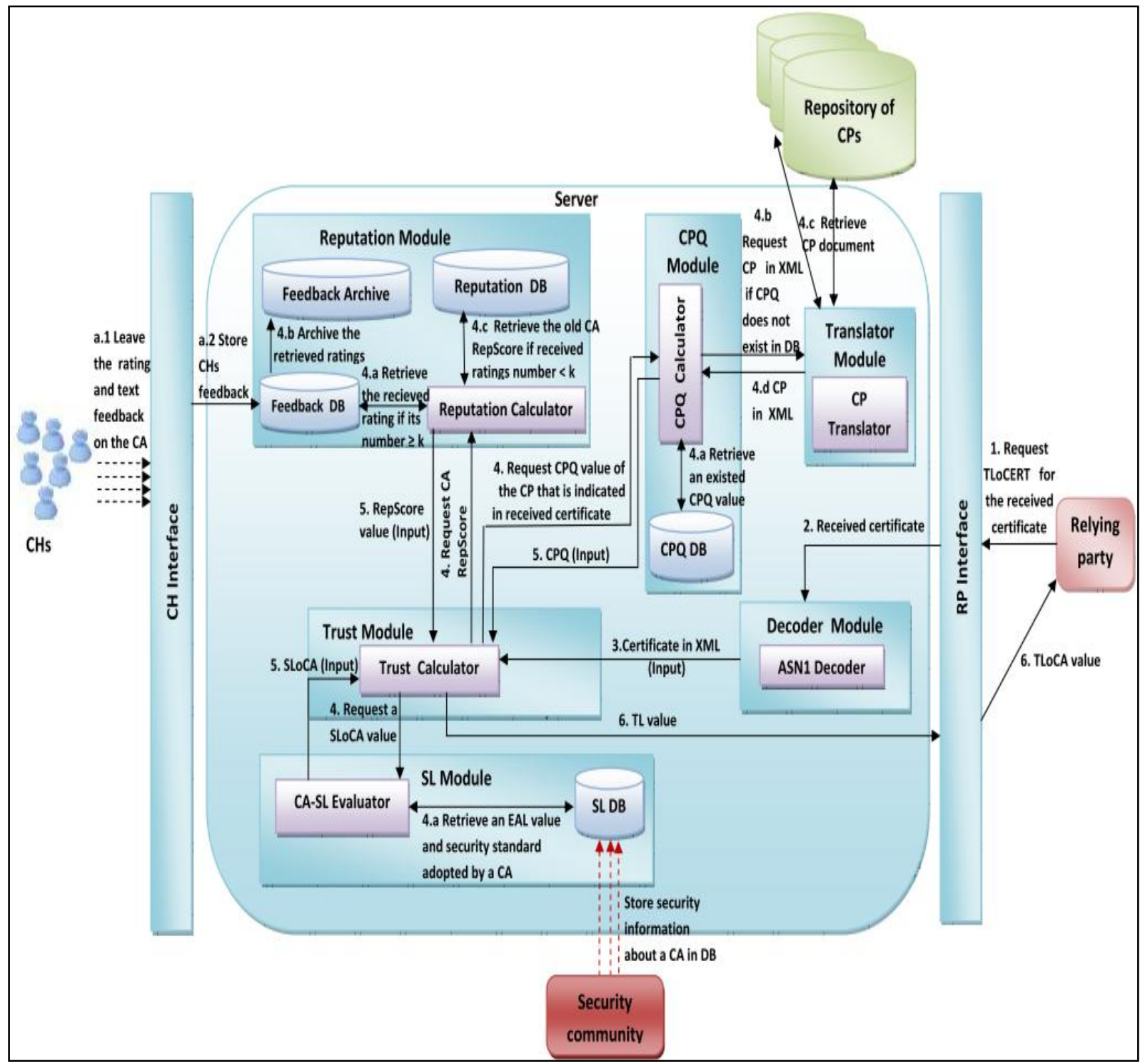

Figure 1. Suggested trust framework architecture.

- Server: It includes six components: the CPQ module, security module, reputation module, decoder module, translator module, and trust module.

- Translator module: On the reception of the CPQ calculator request regarding a $\mathrm{CP}$ in $\mathrm{xml}$ format, the $\mathrm{CP}$ translator searches in CPs repository for extracting the requested $\mathrm{CP}$ and translates it into XML format. The translation process is made according to the rules which will be defined and discussed in a future paper. After generation of the $\mathrm{CP}$ $\mathrm{XML}, \mathrm{CP}$ translator sends it to CPQ calculator in order to use it in CPQ calculation.
CA SL value. This value is then passed to the trust module for computing a CA trust level.

- CPQ Module: It contains two components: CPQ database (CPQ DB) and CPQ calculator. The second computes a CPQ value in applying an algorithm used to fetch information from the XML CP. The calculated value is passed to the trust module and stored in CPQ database

- Trust Module: Its role is to calculate a received certificate TLoCERT value which is requested by a RP. This module contains the trust calculator. It use a parser to analyze a provided X.509 certificate in 
XML format and to extract from it the data elements that are needed for calculating the TLoCERT. This component calculates and evaluates this level based on the calculated TLoCA, the CPQ received from the CPQ module, and the score value of extensions fields content. It is important to note that a TLoCA is computed by the trust calculator on the basis of two parameters: RepScore provided by the reputation module, and the SL value sent by the SL evaluator.

- Reputation module: When $\mathrm{CHs}$ leave their ratings via a $\mathrm{CH}$ interface, these rating are stored in feedback database (DB), and then retrieved by the reputation calculator for calculating/updating the CA RepScore. The used ratings are archived in feedback archiver in order to free the feedback DB space.Before calculating a RepScore value, a reputation calculator checks if a number of the received ratings achieves a $10 \%$ of the computed threshold which will be defined in next section. Finally, the computed RepScore is sent to the trust module for computing a TLoCERT and is stored in Reputation DB.

- Decoder module: It contains an ASN decoder. Note that an X.509v3 certificate structure is presented in general according to the ASN.1 format. This decoder allows the converting of the received X.509v3 certificate from RP into XML format. The generated XML certificate is sent to the trust module.

The CHs can access our suggested framework through a link which is embedded into e-services applications such as e-government, e-commerce. Then, they leave their appreciations about a CA that has granted them a certificate. However, some $\mathrm{CHs}$ may not give their feedback and think that it is not interested. It is needed to provide them the incentives and explain them that the importance of their participation for making decision about a CA trustworthiness in granting a validate certificate. That permits to encourage them to use our framework and explain their point of view on a specific CA.

\section{Evaluating trust in $X .509$ certificate}

Defining the factors that have an influence on making decision about certificate trustworthiness is a main task for computing its trust level. RP trusts a certificate for the correctness of its content based on the procedures announced in the CP/CPS and the CA trustworthiness. Moreover, a negative or positive reputation of CA can have an influence in deciding whether a user will trust or not this authority. On the other hand, the malicious hackers can attack a CA and issues false certificates. Then, its security is an important factor that has an influence on making decision about its trustworthiness. We describe in the following subsections the TLoCert calculation method in details

\subsection{Calculating RoCERT}

At the beginning, we evaluate certificate correctness by checking the contents of two fields which are a key usage and validity using our system. We consider that if the validity period of the certificate is not respected and/or the key usage field does not correspond to the application usage requirements, such certificate must be rejected and it is untrustworthy. In this case, the trust assessment process will be stopped. Then, we send a message to RP in order to inform him that the requested certificate is untrustworthy. The certificate correctness evaluation process is given in the flow diagram on Fig. 2. We evaluate these certificate fields' contents by using a rating mechanism. We consider three ratings (RoCERT) 0, 0.5, 1 which are assigned to the certificate and express its initial correctness evaluation. As explained in Fig. 2, firstly, we verify the key usage field. If its content corresponds to the application usage requirements, we check certificate validity field.

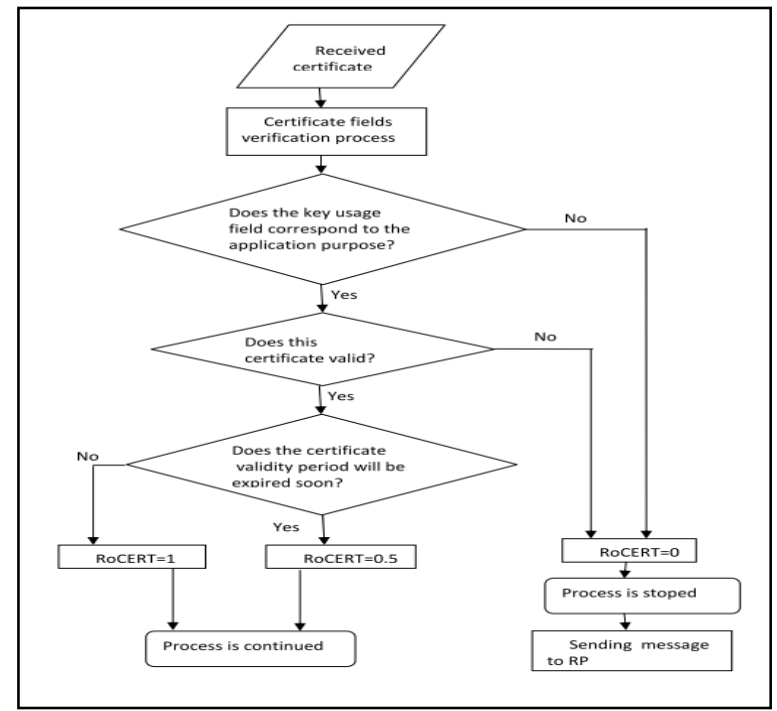

Figure 2. An initiated evaluation of a certificate correctness.

If it is valid, we verify its validity period. When the system found that its validity will be expired soon, the assigned rating is RoCERT $=0.5$. Otherwise, we assign to such certificate a rating RoCERT $=1$. In this case, the system continues with the evaluation process which will be discussed in next subsections. On the other hand, if a certificate is invalid and/or its key usage does not match a required purpose of the application, the given rating is then RoCERT $=0$ and it will be rejected.

\subsection{Determining a CA trust level}

We calculate the TLoCA on basis of two parameters RepScore and SL that are defined as follow: 
5.2.1. Calculating the RepScore. We compute a RepScore on the basis of feedback ratings given by $\mathrm{CHs}$ using the exponentially weighted moving average (EWMA) method [16]. The reason behind using this method is that it permits to weigh new observations more and do not ignore old ones. In this way, when updating a Repscore, the older value is reduced over time and not ignored. In general, the EWMA Control chart is control limits that permits to determine whether a process is in statistical control [17]. In this context, this methodology is usually used to detect the malicious feedback rating [17].

We calculate a CA RepScore by using the following equation:

RepScore $=\alpha^{*} \operatorname{rtg}+(1-\alpha)$ OldRepScore

Where

- RepScore is a computed reputation score

- OldRepScore represents the old reputation score

$-0<\alpha \leq 1$ is a smoothing constant that presents the weight provided to the previous data. Its value depends to the personal experience and preference. In our case, the lower value may have more influence on the old reputation, while the higher value grants more weight to new rating. Then, we must select an appropriate $\alpha$ for controlling the strictness of our system. We suggest that $\alpha$ takes a value between 0.6 and 0.7 .

-rtg presents a new rating provided by a $\mathrm{CH}$. follow:

The upper and lower control limits are defined as

$$
\begin{aligned}
& \mathrm{LCL}=\mu 0-\mathrm{L} * \sigma * \sqrt{\frac{\alpha}{2-\alpha}} \\
& \mathrm{UCL}=? \mu 0+\mathrm{L} * \sigma * \sqrt{\frac{\alpha}{2-\alpha}}
\end{aligned}
$$

Where

- $\mathrm{L}$ takes a value 3 (the 3 -sigma control limits) or is selected by using the Saccucci and Lucas tables (ARL $=370$ )

$-\sigma$ presents the estimated variance calculated from the received ratings (historical data).

- $\mu_{0}$ indicates the historical data mean.

Generally, a statistical anomaly is detected when the values fall outside the UCL or LCL. So, if RepScore value is greater than UCL or less than LCL, the last user rating is considered malicious. Then, this rating will not be taken into consideration in calculation process of RepScore and be ignored. Note that UCL and LCL values are varied depending on the new received ratings. Furthermore, the provided ratings explain the $\mathrm{CHs}$ appreciations for a specific $\mathrm{CA}$.

They are expressed as a percentage belonging to the threshold $[0,100]$, as shown in Table 1 :
Table 1. Explanation of the provided ratings

\begin{tabular}{|l|l|}
\hline \multicolumn{1}{|c|}{ Rating } & \multicolumn{1}{c|}{ Explanation } \\
\hline$[0,20]$ & Very bad \\
\hline$(20,40]$ & Bad \\
\hline$(40,60]$ & Moderate \\
\hline$(60,80]$ & Good \\
\hline$(80,100]$ & Perfect \\
\hline
\end{tabular}

The algorithm1 shows the reputation score calculation. The function "repscore" gets as input the parameters values defined previously for computing the RepScore. Besides, we apply the control chart methodology in our algorithm for detecting the malicious ratings when the participated $\mathrm{CHs}$ number is representative. This means that their number is equal or greater than a threshold value which is determined using a Statistical Sampling Technique (stratified sampling). This threshold value is defined by a sample size $\mathrm{n}$ which represents the total user subpopulation certificed by a same authority. We note that our population is reperesented by the users' total number that utilize e-services applications, into which our platform link is embedded. We divide members of this population into different subgroups (subpopulation) according to name of CA that certifies them. All members of each subgroup have been certified by a same CA. In addition, we calculate a sample size that represents each subpopulation by using the following formula [18]:

$$
\mathrm{n}=\frac{\mathrm{N}}{1+\mathrm{N} * \mathrm{e}^{2}}
$$

Where

$-\mathrm{N}$ presents a size of the user subpopulation and $\mathrm{n}$ is the sample size,

-e is the precision level ( $\mathrm{e}=5 \%)$ which is defined as the range in which the true value of the population is estimated to be [18].

It is important to note that the total number of user population $\mathrm{T}$ is equal to sum the number of user within each subgroup i $\mathrm{T}=\sum \mathrm{Ni}$.

Moreover, the calculated reputation score values belong to the range $[0,1]$. Also, LCL and UCL values would be included in $[0,1]$. The bad reputation is represented by a score including in $(0.2,0.4]$. If the CA reputation is worst, its score is then comprised in $[0,0.2]$. The moderate reputation has a score including in $(0.4,0.6]$. Besides, the good reputation score belongs to $(0.6,0.8]$ whereas perfect reputation score is belonging to $(0.8,1]$.

5.2.2 Evaluating a SL. CA Security is a complex property that cannot be easily measured. CA system is vulnerable for any attack kind. The attacker can generate signed false certificate revocation lists (CRLs). Then, it is needed to protect the CAs systems against security breaches 
by implementing the highest security standards, and ensuring their compliance with the security requirements by a regular audit.

On the other hand, each CA uses software for granting the certificates, generating public/private key pairs, and managing the CRLs. The software security features are assessed by establishing the evaluation assurance level (EAL) if it is certified by Common Criteria Standard (CC). In this context, the EAL level is an important factor for trusting a CA. The CC defines seven levels: EAL1, EAL2, EAL3, EAL4, EAL5, EAL6, and EAL7. The highest assurance level is between [EAL5-EAL7]. The levels EAL1-2 present low assurance. Thus, the levels included in [EAL3EAL4] are medium assurance. Hence, we evaluate a CA system security level on the basis of a combination between the CC level (EAL) provided for software used by this CA and an implemented security standards. We show how we precise SL values in the following Table 2:

Table 2. Explanation of the assigned security maturity level (SL)

\begin{tabular}{|c|l|l|l|}
\hline EAL & \multicolumn{1}{c|}{$\begin{array}{c}\text { Security } \\
\text { standard }\end{array}$} & SL & Explanation \\
\hline [EAL1-EAL2] & - & 0 & Weak \\
\hline [EAL1-EAL2] & ISO/IEC 27001 & 0.5 & Medium \\
\hline [EAL3-EAL4] & - & 0.5 & Medium \\
\hline [EAL3-EAL4] & ISO/IEC 27001 & 1 & Strong \\
\hline [EAL5- EAL7] & - & 1 & Strong \\
\hline [EAL5- EAL7] & ISO/IEC 27001 & 1 & Strong \\
\hline
\end{tabular}

The SL values are 1,0.5, and 0 that correspond to strong, medium, and weak respectively. For instance, we assign $\mathrm{SL}=0$ to a $\mathrm{CA}$ that do not adopt any security standard and the used software EAL is one of these levels EAL1, EAL1+ or EAL2. Besides, a SL value is updated when an implemented security standard or EAL is changed.

5.2.3 Determining TLoCA. TLOCA is quantitative information which indicates the CA trustworthiness. Its value depends on RepScore and SL. We compute it using the weighted average method, as shown in the following equation:

$$
\text { TLoCA }=\frac{w 1 * \text { RepScore }+\mathrm{w} 2 * \text { SL }}{\sum_{i=1}^{2} \text { wi }}
$$

Where w1, w2: the assigned Weights and $\sum_{\mathrm{i}=1}^{2}$ wi equals to 1 . These weights specify the impact of these factors on the TLoCA.

A new TLoCA is computed each time the aforementioned parameters are modifed. RepScore is updated when a new received ratings number is equal or more than $10 \%$ of the threshold whichi is calculated using an equation 4 or the RP request about a CA reputation. SL seems to be constant. However, it can be updated when the security standard and the EAL level change. Consequently, the weight adjustment is determined according to

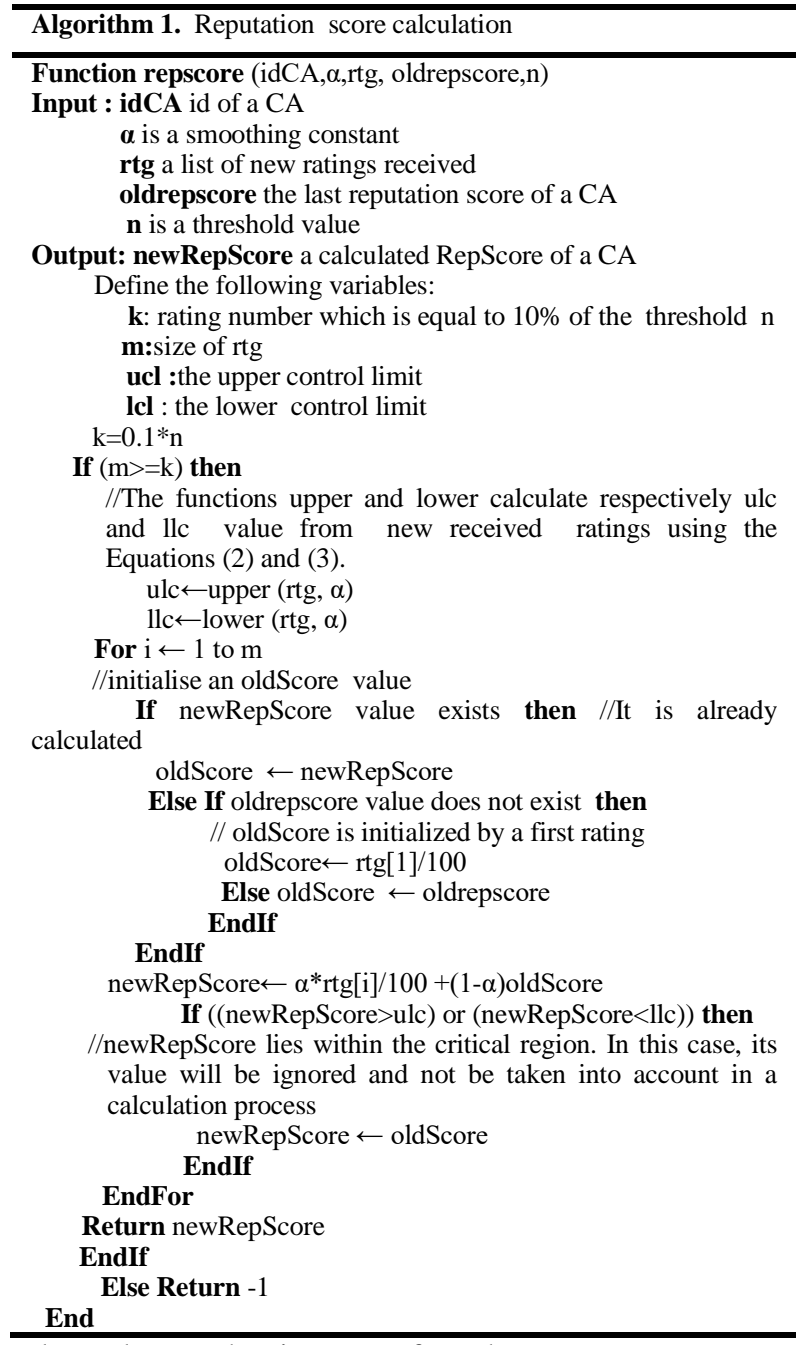

depends on the impact of each parameter on TL computation process. It is described in the following steps:

- Step 1: Initially, we may not get any rating from CHs. Then, the TLoCA is initialized by the SL value using an equation 7 , as follow:

$$
\text { TLoCA }=\text { SL }
$$

- Step 2: In this step, few CHs may utilize our framework and leave their appreciation for a specific CA that has been issue them certificates. In this case, the CA reputation can not be judged on the basis of the low number of CHs. As a result, we assign to RepScore the weight 0.25 which must be lower than that of SL.

- Step 3: When CHs number grows and achieves a threshold value, we augment the weight value of RepScore $\left(\mathrm{w}_{1}=0.75\right)$. It is higher than that of SL.

The algorithm 2 presents the TLoCA calculation. The computed level would be included in a threshold $[0,1]$. 


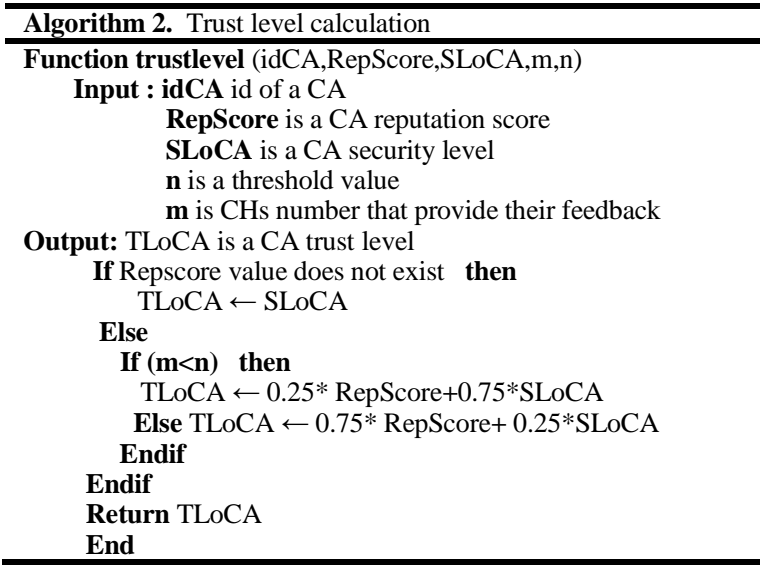

There are four trust levels: high trust, medium trust, low trust, and no trust. The high trust level is included in $(0.75,1]$, whereas the minimal value representing the case of "no trust" belongs to the range $[0,0.25]$. If a trust level is low, its related value is included in $(0.25,0.5]$. Finally, the medium trust level is represented by the values including in $(0.5,0.75]$.

\subsection{Calculating the CPQ}

Each CA issues a certificate based on its CP/CPS which describes the set of rules maintained by the CA during the certificate life cycle. $\mathrm{CP}$ is then an important document to evaluate the certificate trust level. It may be written by a language different from the RP's language and very long. Thus, it can contain the difficult terms that RPs do not understand them. For these reasons, it is difficult for RPs to judge it. In fact, it is needed to automate the CP interpretation process which can help RPs to make trust decision about a received certificate.

In this paper, we present an automated process for evaluating the procedures described in the published CP. In fact, we present an algorithm for calculating a CPQ which indicates that these indicated procedures are weak or rigorous. Firstly, we represent CP document, which is based on a RFC 3647 template standard, in digital form. The more a policy is formalized, the more the evaluation process is easily achieved by an automatic machine [20]. We translate then it into XML format in order to represent it as tree structure which is described in RFC 3647, manipulate it easily and parse it for calculating a CPQ value (see Figure 2). The translation process is made according to the rules that will be discussed in a future paper.

The formal presentation of $\mathrm{CP}$ is defined and detailed in the Internet RFC3647 [19]. This presentation is not standard but it is broadly utilized by all the Internet Community [20]. Therefore, we use its structure for $\mathrm{CP}$ formalization. The nine primary components suggested by RFC 3647 [19] are show as follow:
1)Introduction
2)Publication and repository

3)Identification and Authentication 4)Certificate
Life-Cycle Operational Requirements 5)Facilities, Management, and Operational controls 6)Technical Security Controls 7)Certificate, CRL, and OCSP Profile 8)Compliance Audit 9)Other business and Legal Matters

The proposed XML format of a CP is as follows:

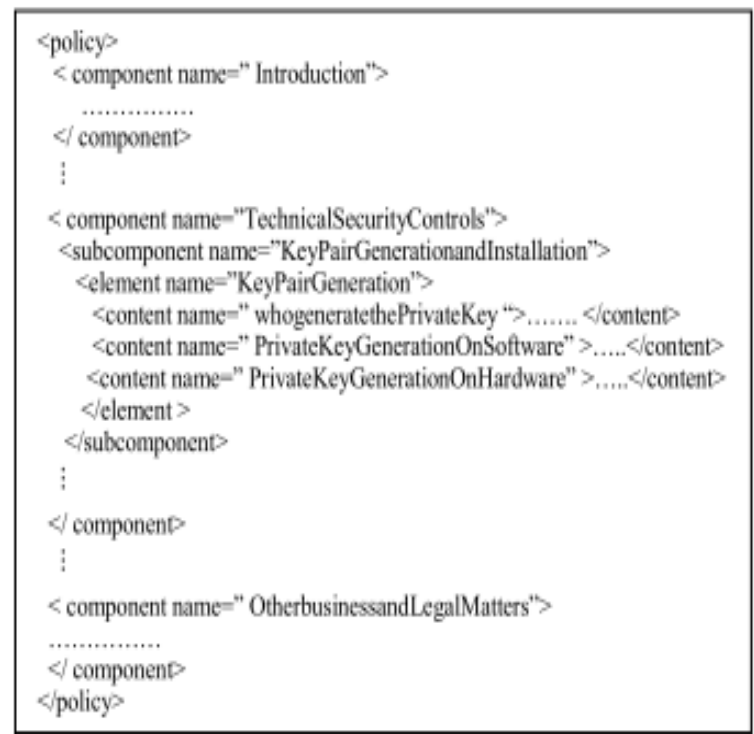

Figure 3: Proposed XML CP

Within the framework of the RFC 3647, a CP composes of components, which can be contained multiple subcomponents, and a subcomponent may comprise several elements.

For instance, the component 'Technical Security Controls' composes of the eight subcomponents: 'key pair generation and installation', 'private key protection and cryptographic module engineering controls', 'time-stamping', etc. As illustrated in Figure 2, the subcomponent 'key pair generation and installation' consists of several elements. Each element may comprise multiple contents.

The algorithm 3 presents a calculation process of CPQ value. It takes a XML CP file as input which is parsed using a DOM parser. This parser models it in a tree structure (XML DOM tree) for manipulating it easily. First, we extract its root element and use the function 'getchildren' as explained in algorithm 4 to obtain its children nodes. Then, we traverse XML DOM tree a node by node in order to extract from it the information needed to calculate a CPQ value automatically.

In addition, we calculate a score of each component using the function 'score comp' explained in algorithm 5. We sum all the computed score values and divide the result on the component number:

$$
\mathrm{CPQ}=\sum_{\mathrm{i}=1}^{9} \text { scorei } / 9
$$




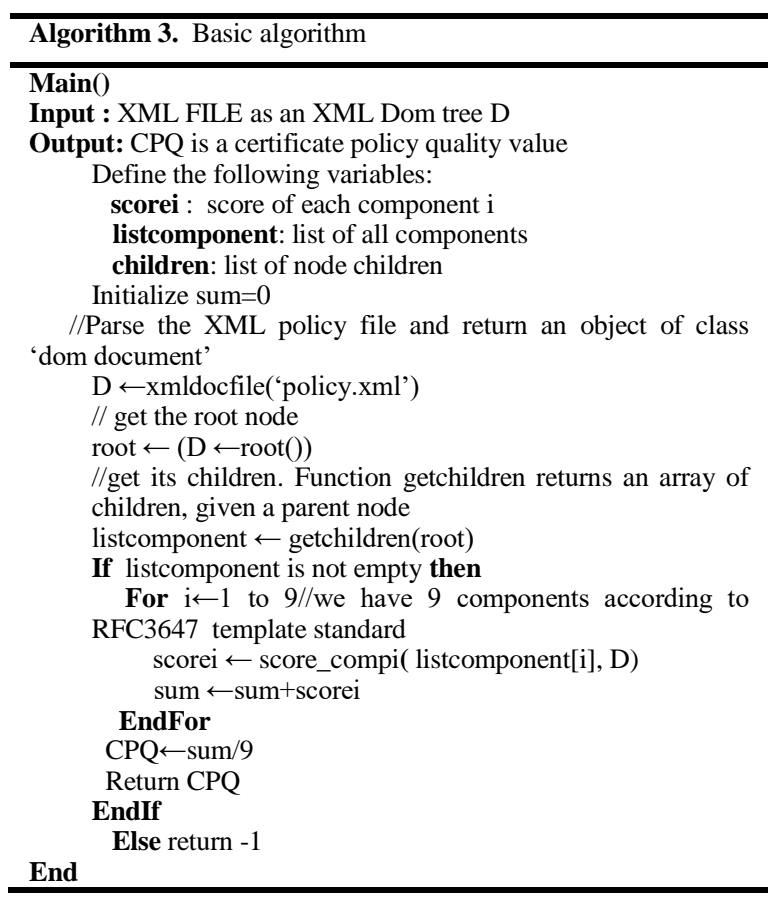

The function 'getchildren' in algorithm 4 take a parent node as input and return its children which are element type.

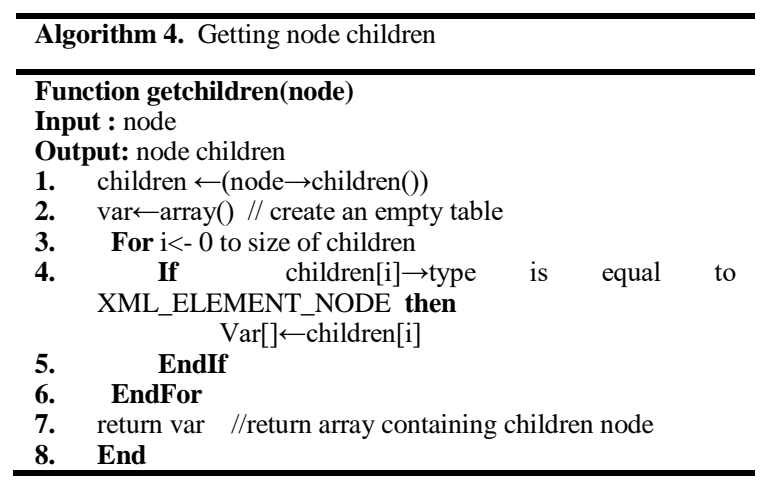

As mentioned previously, the component score is calculated by using the function 'score comp' which differ from a component to other depending on its content. We present below an algorithm 5 defining the function 'score_comp1' that calculate a score of the component 'Technical Security Controls'. It takes a component name and XML DOM tree as input. Then, we get the children of this component and traverse them node by node for computing the score value using a scoring method. We assign a score to each content of element (cont_score) according to its importance in computing the TLoCERT using a function 'score_content'. The assigned score takes a value $0,0.5$ or 1 presents respectively low, medium, and high. It explains the importance of each content for calculating a TLoCERT. Then, we compute a score of each element belongs to a specific subcomponent (elmt_score) by summing all the scores values of their contents and divide the result on the number of these contents using a function 'score'. Next, we use a same function 'score' to calculate score of each subcomponent (subcomp_score) by summing all the scores values of their elements and divide the result on the number of these elements. We use a same process for calculating a component score (comp_score); we sum all the score values of its subcomponents and divide the result on a number of these subcomponents (see Algorithm 4). For example, for the element 'Key Pair Generation' of the subcomponent 'Key pair generation and installation", if a key pair is generated in hardware, an assigned score to this element is 1. A key pair generated in hardware is more trustworthy than that generated in software.

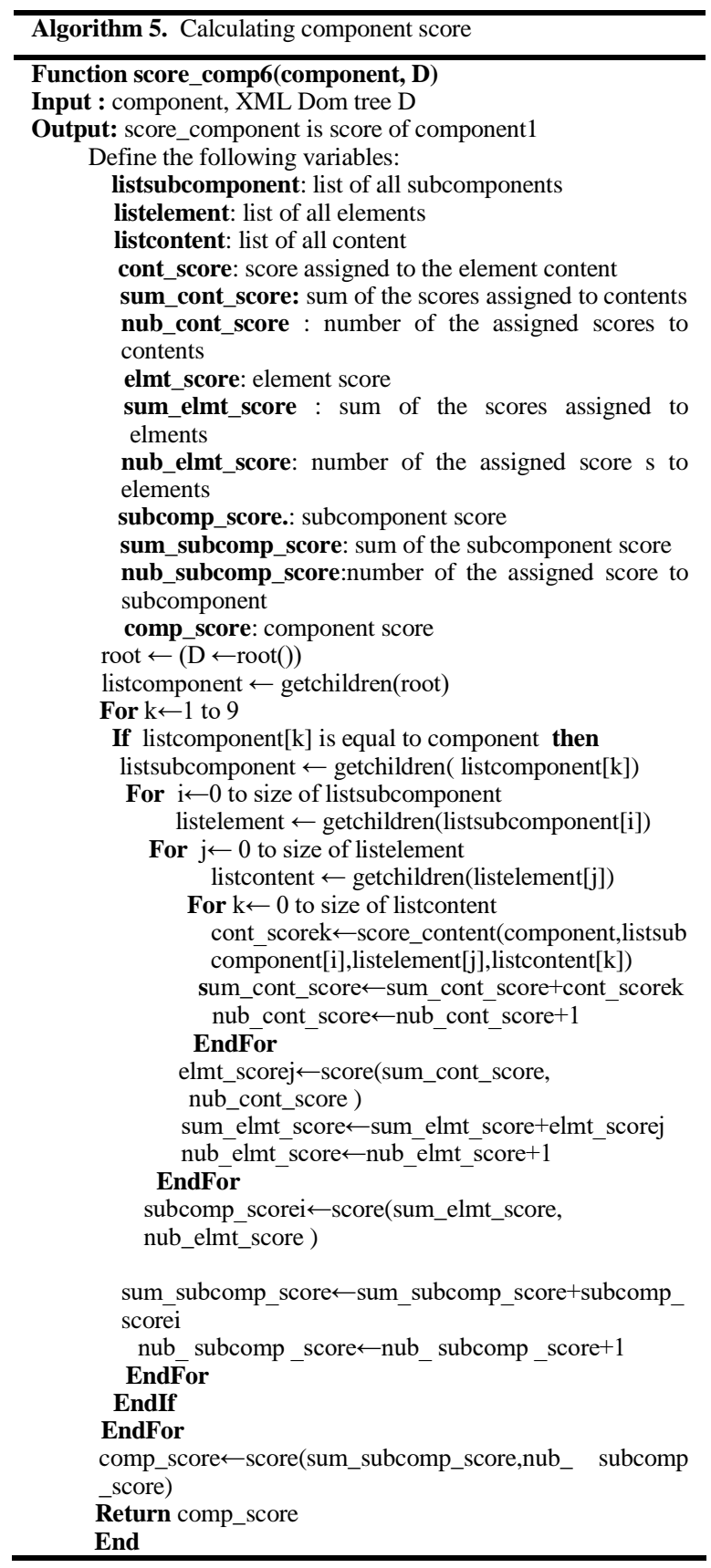

We note that the functions 'score', and 'score_content' will be described in a future paper. 


\subsection{Calculating the TLoCERT}

As mentioned previously, computing TLoCERT is based on three parameters that are the CPQ, TLoCA, and RoCERT.We calculate it using the weighted average method, as given in the following equation:

$$
\text { TLoCERT }=\frac{\mathrm{w} 1 * \text { RoCERT }+\mathrm{w} 2 * \mathrm{TLoCA}+\mathrm{w} 3 * \mathrm{CPQ}}{\sum_{\mathrm{i}=1}^{3} \mathrm{wi}_{\mathrm{i}}}
$$

Where $\mathrm{w} 1=0.2, \mathrm{w} 2=0.4$, and $\mathrm{w} 3=0.4$ are the assigned weights and $\sum$ wi equals to 1 . These weights define the impact of these parameters on the TLOCERT calculation. In addition, the aforementioned parameters are taken as input of the TLoCERT calculation algorithm 6 .

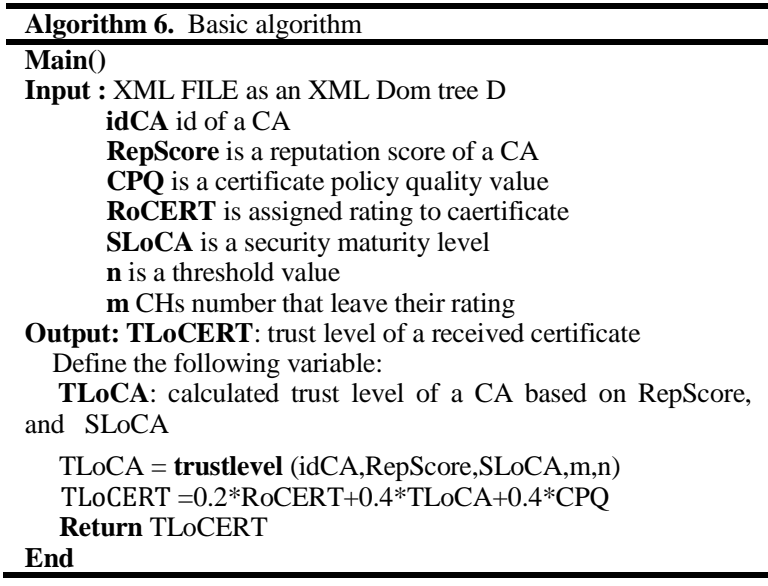

The computed trust level is included in $[0,1]$. There are four trust levels: no trust, low trust, medium trust, and high trust. The first level belongs to the range $[0,0.25]$, whereas the second is included in $(0.25,0.5]$. The high trust is represented by a level belonging to $(0.75,1]$. Finally, the medium trust has a level included in $(0.5,0.75]$.

\section{Case study}

In this section we present an example of scenario that explain how certificate trust level can be calculated for helping RP in making decision about its trustworthiness. Then, we consider the scenario as follow:

- CA issues certificate for its $\mathrm{CH}$ in order to use it for confirming its identity. When RP receives this certificate, he needs to check its trustworthiness. Then, he accesses our system and requests its TLOCERT value in order to decide whether accept or not a received certificate.

At initial, we propose that the issuer CA implements a security standard ISO/IEC 27001 and the CC level of its software is EAL3. According to the table 3 , the SLoCA is equal to 1 . Also, the obtained CPQ is equal to 0.7 and RoCERT takes a value 1 . We use a weighting factor $\alpha=0.6$ in RepScore computation.
We suggest that a number of $\mathrm{CHs}$, that utilize our platform and are certified by a CA in question, achieves 400. At first, we check whether this number achieves a threshold value or not. As indicated previously, a threshold takes a value of a sample size $\mathrm{n}$ which present the total user subpopulation. In our example, we consider that the users number that utilize e-services application, into which our platform link is embedded, is 70000 users. We classify this population into subgroups in accordance with a CA name. In this scenario, we take into consideration members of subgroup that are certified by the CA in question. Its related number is 5000. Moreover, the threshold value is computed by using the following Equation 4:

$$
\mathrm{n}=\frac{\mathrm{N}}{1+\mathrm{N} * \mathrm{e}^{2}}=\frac{5000}{1+5000 * 0.05^{2}}=370
$$

We conclude that the $\mathrm{CHs}$ number is more than the threshold value $\mathrm{n}$. Then, the weight value assigned to RepScore is 0.75 . Also, 380 users is superior of $10 \%$ of the threshold $\mathrm{n}(0.1 * \mathrm{~N}=37)$. In this case, we calculate LCL and UCL from 370 new received ratings by applying the Equations (2) and (3). We note that the estimated variance $\sigma$ is 0.03 and $\mu_{0}=$ 0.6. Then, we get the result as follow:

$$
\begin{aligned}
& \mathrm{LCL}=0.6-3 * 0.04 * \sqrt{\frac{0.6}{2-0.6}}=0.52 \\
& \mathrm{UCL}=0.6+3 * 0.04 * \sqrt{\frac{0.6}{2-0.6}}=0.68
\end{aligned}
$$

By using the algorithm1, we compute a RepScore value. If its value is outside 0.68 or 0.45 , the last user rating that is utilized for calculating this RepScore will not be taken into account in a calculation process. As a result, we get RepScore $=0.61$.

By applying the algorithm 2, we compute the following TLoCA value:

$$
\mathrm{TLoCA}=0.75 * 0.61+0.25 * 1=0.7
$$

0.7 is comprised in $(0.6,0.8]$. Then, the trust level of the $\mathrm{CA}$ in question is good.

Let's compute a TLoCERT value by applying the Equation (8):

\section{TLoCERT $=0.2 *$ RoCERT $+0.4 *$ TloCA $+0.4 * \mathrm{CP}=0.76$}

0.76 belongs to $(0.75,1]$. As a result, the trust level of the received certificate is high.

\section{Conclusion}

In this paper, we have proposed an approach for calculating certificate trust level. Our solution aims to help RP in making decision concerning a trustworthiness of a received certificate.

In future work, we aim to develop the TLoCA computation process taking into consideration the 
certificates obtained by a CA that we measure its trustworthiness. According to [15], granting a certificate by a trustworthy $\mathrm{CA}$ for another CA implicates that the issuer CA places certain trust in it. In this sense, we will assess this implicit trust in accordance with the issued certificate extensions fields, which will be discussed in detail in a future paper. Thus, we evaluate CA trustworthiness depending on their certificates issued by the other trusted CAs. This new trust evaluation process for a CA will be added to the previous one. That will improve the TLOCA computation when getting a low number of $\mathrm{CHs}$. On the other hand, we will describe in detail a translation process of $\mathrm{CP}$ document into XML format in a future paper. Finally, we will improve our approach and assess its effectiveness in our experimental.

\section{References}

[1] I.Symeonidis, F.Beato, and B. Preneel, "fRiendTrust: A Privacy Preserving Reputation System for Online Social Networks", in Proceedings of the IFIP Information and Communication Technology, Springer-Verlag, 2014, p.17.

[2] A. Jøsang,R.Ismail, and C.Boyd, "A Survey of Trust and Reputation Systems for Online Service Provision", Decision Support Systems journal, Elsevier Science, March 2007, pp. 618-644,

[3] C.Cheshire, "Online Trust, Trustworthiness, or Assurance?", Daedalus, MIT Press Journals, Septembre 2011, pp. 49-58

[4] J.Audun, "PKI trust models", Theory and Practice of Cryptography Solutions for Secure Information Systems, IGI Global, 2013, pp. 279-301

[5] A. Jøsang, "The right type of trust for distributed systems", in Proceedings of the New Security Paradigms Workshop (NSPW), ACM, 1996.

[6] L.Mui,M.Mohtashemi, A.Halberstadt, "A computational model of trust and reputation", In Proceedings of the 35th Hawaii International Conference on System Sciences, 2002, pp. 24312439.

[7] R.C.Mayer, J. H.Davis, and \& F.D.Schoorman, "An integrative model of organizational trust", Academy of Management Review, Academy of Management, vol.20,1995, pp. 709-734.

[8] A.Josang, "Trust and Reputation Systems", Foundations of Security Analysis and Design IV (FOSAD),Springer Berlin Heidelberg, Bertinoro, Italy, September 2007, pp. 209-245.

[9] H. Jingwei, and N. David. "A calculus of trust and its application to PKI and identity management," In IDtrust, ACM New York, 2009, pp.23-27.

[10] C.Jiska, B.Johannes, V.Florian, H. Matthias, and M .Max,"A Distributed Reputation System for Certification Authority Trust Management," 14th IEEE International Conference on Trust, Security and Privacy in Computing and Comm unications (IEEE UbiSafe Symposium), Finland, 2015,pp.1349- 1356.

[11] J.Braun, F.Volk, J.Buchmann, and M.Mühlhäuser, "Trust views for the web PKI," In Public Key Infrastructures, Services and Applications, Springer, 2014,Vol 8341, pp.134-15.

[12] V.Hawanna, V. Y. Kulkarni, R. A. Rane, P. Mestri, S. Panchal," Risk Rating System of X.509 Certificates", $12^{\text {th }}$ International Multi-Conference on Information Processing-2016 (IMCIP-2016), Bangalore, India,2016,pp.152-161.
[13] S.Anooshiravan, M.U.Janjua, N.Porter, P. Hallin, H.Li, X.Su, K.Yiu, A.P.Penta, V.D.Bakalov, B.M.Nitta," Advising Clients about Certificate Author ity Trust," patent application at Politics \& Government Week, February 25, 2016.

[14] L.Gonc A.Martins and R.F.Custodio, "Implementatio $\mathrm{n}$ of Trust Metrics in X.509 Public Key Infrastructure", The Seventh International Conference on Emerging Security Information, Systems and Technologies,Barcelona, SpainAugust 2013, pp.25 31.

[15] H.El Bakkali and B.I. Kaitouni, "A predicate calculus logic for the PKI trust model analysis", IEEE International Symposium on Network Computing and Applications, IEEE, 2001, pp.368 371 ,

[16] J.M.Lucas and M.S.Saccucci, "Exponentially weighted moving average control schemes:properties and enhancements", Technometrics, Taylor \& Francis, Ltd., USA, 1990, vol. 32, pp.1-12.

[17] V.V.Rajendran, and S.Swamynathan, "Hybrid model for dynamic evaluation of trust in cloud services," Wireless Networks, Springer, 2015, pp. 1-12,

[18] D.I.Glen,(1992) "Determining sample size", http://www.sut.ac.th/im/data/read6.pdf

[19] S.Chokhani, W.Ford, R.Sabett, C.Merrill, and S.Wu, "RFC 3647: internet X.509 public key infrastructure certificate policy and certification practices framework", Nov 2003.

[20] V. Casola, A. Mazzeo, N. Mazzocca and M. Rak, "An innovative policy-based cross certification methodology for public key infrastructures", Public Key Infrastructure, Springer, 2005. 